\title{
A novel iterative reconstruction algorithm allows reduced dose multidetector-row CT imaging of mechanical prosthetic heart valves
}

\author{
Jesse Habets • Petr Symersky • \\ Bas A. J. M. de Mol • Willem P. Th. M. Mali • \\ Tim Leiner $\cdot$ Ricardo P. J. Budde
}

Received: 2 May 2011/Accepted: 30 September 2011 / Published online: 15 October 2011

(C) The Author(s) 2011. This article is published with open access at Springerlink.com

\begin{abstract}
Multidetector-row CT is promising for prosthetic heart valve (PHV) assessment but retrospectively ECG-gated scanning has a considerable radiation dose. Recently introduced iterative reconstruction (IR) algorithms may enable radiation dose reduction with retained image quality. Furthermore, PHV image quality on the CT scan mainly depends on extent of PHV artifacts. IR may decrease streak artifacts. We compared image noise and artifact volumes in scans of mechanical PHVs reconstructed with conventional filtered back projection (FBP) to lower dose scans reconstructed with IR. Four different PHVs (St. Jude, Carbomedics, ON-X and Medtronic Hall) were scanned in a pulsatile in vitro model. Ten retrospectively ECG-gated CT scans were performed of each PHV at $120 \mathrm{kV}, 600 \mathrm{mAs}$ (high-dose $\mathrm{CTDI}_{\mathrm{vol}}$ $35.3 \mathrm{mGy}$ ) and $120 \mathrm{kV}, 300 \mathrm{mAs}$ (low-dose CTDI $\mathrm{vol}_{\mathrm{v}}$ $17.7 \mathrm{mGy}$ ) on a 64 detector-row scanner. Diastolic and systolic images were reconstructed with FBP (high and low-dose) and the IR algorithm (low-dose only). Hypoand hyperdense artifact volumes were determined
\end{abstract}

J. Habets $(\bowtie) \cdot$ W. P. Th. M. Mali · T. Leiner .

R. P. J. Budde

Department of Radiology, University Medical Center

Utrecht, P.O. Box 85500, E01.132, 3508 GA Utrecht,

The Netherlands

e-mail: J.Habets@umcutrecht.nl

P. Symersky · B. A. J. M. de Mol

Department of Cardiothoracic Surgery, Academic

Medical Center, Amsterdam, The Netherlands using two threshold filters. Image noise was measured. Mean hypo- and hyperdense artifact volumes $\left(\mathrm{mm}^{3}\right)$ were 1,235/5,346 (high-dose FBP); 2,405/6,877 (lowdose FBP) and 1,218/5,333 (low-dose IR). Low-dose IR reconstructions had similar image noise compared to high-dose FBP $(16.5 \pm 1.7$ vs. $16.3 \pm 1.6$, mean $\pm \mathrm{SD}$, respectively, $P=1.0$ ). IR allows ECGgated PHV imaging with similar image noise and PHV artifacts at $50 \%$ less dose compared to conventional FBP in an pulsatile in vitro model.

Keywords Prosthetic heart valve . Computed tomography $\cdot$ Iterative reconstruction . Artifacts $\cdot$ Image noise $\cdot$ Reconstruction algorithm

\section{Introduction}

Multidetector-row CT (MDCT) is a promising imaging technique for prosthetic heart valve (PHV) assessment. It provides complementary information to echocardiography and fluoroscopy in patients with suspected PHV dysfunction [1-5]. MDCT scanning of PHVs has been mainly performed with retrospective ECG-gated scan protocols which enable dynamic PHV leaflet assessment and imaging of periprosthetic anatomy in both systole and diastole [6]. In an effort to minimize PHV-related artifacts and to improve image quality, some authors have adjusted exposure parameters by increasing either tube voltage $(140 \mathrm{kV})$ [7] or tube current (800-1,000 mAs) [3]. However, these 
adjustments cause an increase in radiation exposure, which is already substantial with retrospective ECGgating (up to $20 \mathrm{mSv}$ ) [3, 7]. For the use of MDCT in clinical practice, it is important to reduce radiation dose while retaining the ability to assess PHV leaflet motion and anatomy in systole and diastole.

Currently, MDCT uses filtered back projection (FBP) for image reconstruction from raw data [8]. Alternative iterative image reconstruction algorithms have been available for a long time and used in positron emission tomography (PET) and single photon emission computed tomography (SPECT) [9, 10]. Recently, technical developments and increased computational power have allowed the use of novel iterative reconstruction (IR) algorithms for multislice CT image reconstruction $[8,11,12]$. Theoretically these new reconstruction algorithms allow imaging at reduced dose with retained image quality [8]. Furthermore, these new reconstruction algorithms may reduce PHV artifacts as well. At present, it is unknown whether application of these iterative reconstruction algorithms in MDCT scans of PHVs is beneficial for reduction of radiation dose and PHV induced artifacts.

The purpose of the current study was to assess whether IR allows PHV imaging with reduced radiation dose without increased image noise. In addition, we assessed the effect of using IR on the presence and extent of hypo- and hyperdense PHV artifacts in both systolic and diastolic phases.

\section{Methods}

Valves

Four different mechanical PHVs including three bileaflet valves: St Jude (SJ, St Jude Medical Inc., St Paul MN, $27 \mathrm{~mm}$ ), Carbomedics (CM, Carbomedics Inc., Austin, TX, $21 \mathrm{~mm})$, ON-X (ON-X, ON-X Life Technologies Inc., Austin, TX, $27 \mathrm{~mm}$ ) and one tilting disc valve: Medtronic Hall (MH, Medtronic Inc., Minneapolis MN, $27 \mathrm{~mm}$ ) were inserted in a pulsatile in vitro model which was described in detail before [13]. In brief, this model consists of a pump-driven mock loop connected to a custom-made polymethyl methacrylate (PMMA) valve chamber via an inlet and outlet portion. The valve chamber used in this study housed a central mounting ring placed under a 45-degree angle to the CT gantry to simulate the

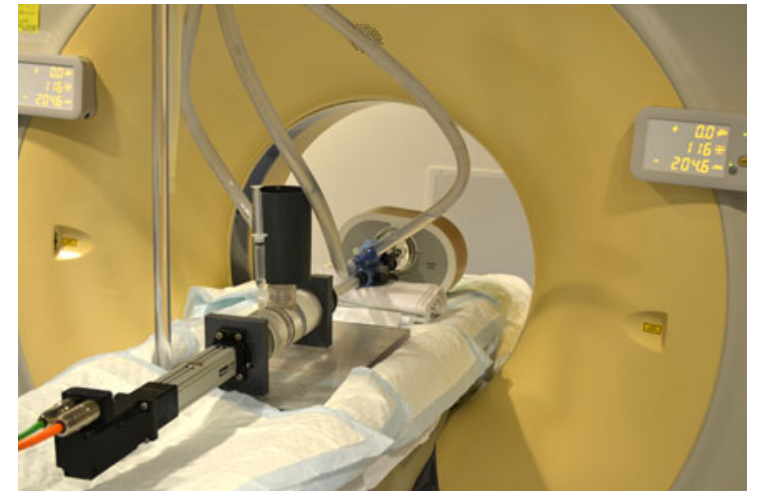

Fig. 1 Photograph of in vitro pulsatile model in the 64 slice CTscanner (Brilliance 64, Philips Medical Systems, Cleveland, Ohio)

normal in vivo aortic position. The different PHVs were inserted in this mounting ring. The valve chamber was placed in a commercially available anthromorphic thoracic phantom (QRM GmbH, Möhrendorf, Germany) to simulate radiation absorption and scattering from the human thorax. A computer controlled piston-pump produced 60 pulses per minute and emitted an artificial ECG signal, which was read by the CT scanner. Water was used for perfusion in this study. No contrast administration was performed. The valve chamber within the thoracic phantom was positioned in the 64 detector-row CT scanner (Fig. 1).

\section{CT scanner}

For each PHV type, twenty scans were performed: ten at $120 \mathrm{kV}, 600 \mathrm{mAs}$ (high dose) and ten at $120 \mathrm{kV}$, $300 \mathrm{mAs}$ (low dose). Scanning was performed on a 64 detector-row CT scanner (Brilliance 64, Philips Medical Systems, Cleveland, Ohio). An additional 15 scans at both scan settings were performed of the $\mathrm{St}$ Jude PHV for image noise measurements. Scanning parameters were based on the clinical scanning protocol for retrospectively ECG-gated MDCT coronary angiography: $64 \times 0.625 \mathrm{~mm}$ collimation, 0.42 s rotation time, pitch of 0.2 and a matrix size of $512 \times 512$ pixels. Scan length was identical for each scan at $100 \mathrm{~mm}$. Total DLP and CTDI ${ }_{\mathrm{vol}}$ for each scan as displayed on the scanner console were recorded.

Image reconstruction

Images of $0.9 \mathrm{~mm}$ thickness with $0.45 \mathrm{~mm}$ increment were reconstructed using a cardiac B filter at two phases 

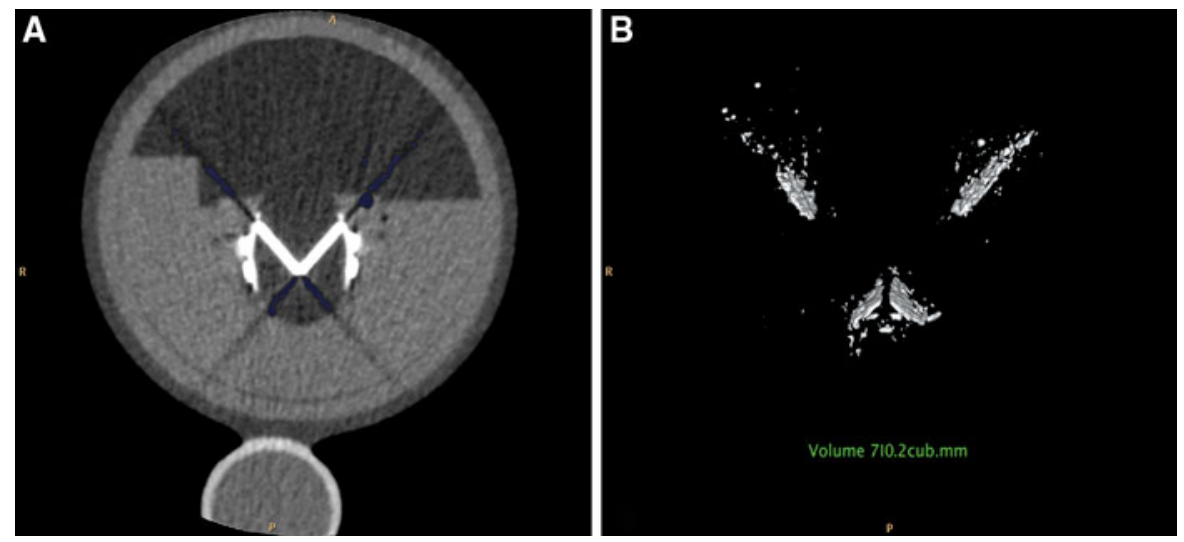

Fig. 2 MDCT image of ON-X Bileaflet PHV. a MDCT image with measured artifacts (blue color), b Frontal plane with 3D volume PHV hypodense artifacts including PHV artifact measurement

of the ECG interval corresponding to diastolic (closed valve) and systolic (open valve) phases. For the highdose scans $(120 \mathrm{kV}, 600 \mathrm{mAs})$, raw data were reconstructed using standard FBP. The IR (iDose, Philips Healthcare, Best, The Netherlands) applies a maximum likelihood denoising algorithm based on Poisson statistics on the raw projection data. Subsequently, reconstructed images are compared to optimal anatomical structures in image space, allowing noise reduction without altering the characteristics and overall appearance of the initial image. Besides noise reduction, iDose may decrease streak artifacts. iDose allows reconstruction of images at seven different levels with level one having the least influence on image reconstruction and level seven having most influence.

From the raw data of the low-dose scans performed at $120 \mathrm{kV}, 300 \mathrm{mAs}$ two different image sets were reconstructed: one image set using standard FBP and one using level 4 IR. Level 4 iDose theoretically leads to image denoising by $29 \%$. A dose reduction from 600 to $300 \mathrm{mAs}$ (factor 2 decrease) would theoretically result in a noise increase by a factor $1.41(\sqrt{ } 2)$ [14]. Applying level 4 iDose would theoretically reduce noise in the low-dose (300 mAs) images to the level of the high-dose scans (noise increase by factor 1.41 due to lower mAs setting, and subsequent reduction by $29 \%$ due to IR).

Image analysis and artifact measurement

Images were transferred to a dedicated workstation (Extended Brilliance Workstation, Philips Medical
Systems, Philips, Best, The Netherlands) for analysis. On the workstation three-dimensional volumerendered images were reconstructed from which the thoracic phantom was digitally excised. Subsequently, volumetric threshold filters were used for measurement of hyper- and hypodense artifacts emanating from the PHV (Fig. 2). For hypodense artifacts, the upper limit of the threshold value was established by measuring the Hounsfield Unit (HU) values of the least dense periprosthetic structure (water) and subtracting approximately 3 times the standard deviation (SD) of the CT water density. The threshold for hyperdense artifacts was derived from the CT density of the surrounding PMMA structure and adding approximately 3 times the SD of the HU measurement. According to a method described elsewhere [15], threshold filters were made to include all voxels below the threshold for the hypodense artifacts and above the threshold for the hyperdense artifacts. Thresholds used in this experiment were set at $\leq 50$ and $\geq 175$ HU for hypoand hyperdense artifacts, respectively. At these settings the $\leq 50 \mathrm{HU}$ filter measures the volume of hypodense artifacts, and the $\geq 175 \mathrm{HU}$ filter measures the volume of hyperdense artifacts, including the PHV ring and leaflets.

Image noise (SD of the HU measurement) was measured in the 25 scans of the St Jude PHV using a circular region of interest (ROI, diameter $10 \mathrm{~mm}$ ) in a homogenous section of the PMMA structure in the valve chamber on identical locations in the different reconstructions (Fig. 3). 


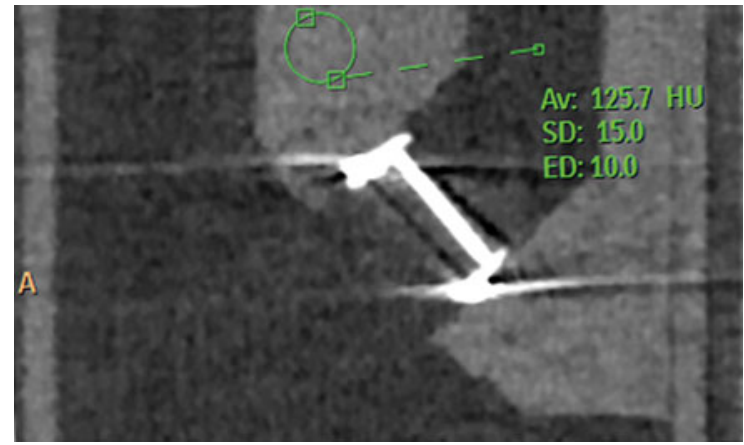

Fig. 3 MDCT image of St Jude bileaflet PHV. Image noise (SD) is measured with a circular region of interest in a homogenous part of the polymethyl methacrylate (PMMA) structure. $A v$ (average image signal), $H U$ Hounsfield Units, $E D$ external diameter $(\mathrm{mm})$

Data analysis

Data were analyzed using SPSS software (SPSS Statistics Version 15.0, SPSS Inc, Chicago, IL) and presented as means \pm SD. For each PHV type, a twoway repeated measures analysis was performed with reconstruction algorithm $(120 \mathrm{kV}, 600 \mathrm{mAs} F B P$; and $120 \mathrm{kV}, 300 \mathrm{mAs}$ FBP and $120 \mathrm{kV}, 300 \mathrm{mAs}$ iDose level 4) and reconstruction phase (systolic or diastolic) defined as within-subject factors. Post-hoc pairwise testing with Bonferroni correction was performed to compare different reconstruction algorithms and reconstruction phases. Mean image noise was compared for the different reconstruction algorithms in a repeated measures analysis. Statistical significance was defined as a $P$ value $<0.05$.

\section{Results}

Dose reduction

For all low-dose scans, CTDI ${ }_{\mathrm{vol}}$ and DLP were equal (17.7 and $236.5 \mathrm{mGy}$, respectively). For the high-dose scans, CTDI ${ }_{\mathrm{vol}}$ and DLP were 35.3 and $473.4 \mathrm{mGy}$ respectively and equal for all scans. Radiation dose for low-dose scans was $50 \%$ of high-dose scans.

Image noise

Mean image noise in the high-dose $(120 \mathrm{kV}$, $600 \mathrm{mAs})$ scans $(16.3 \pm 1.6 \mathrm{HU})$ was $29.7 \%$ lower compared to the low-dose $(120 \mathrm{kV}, 300 \mathrm{mAs})$ scans $(23.2 \pm 2.3 \mathrm{HU})$ reconstructed with FBP. The difference was statistically significantly $(P<0.001)$. Low-dose scans reconstructed with iDose level 4 demonstrated similar image noise compared to highdose scans reconstructed with FBP $(16.5 \pm 1.7 \mathrm{HU}$ vs. $16.3 \pm 1.6 \mathrm{HU}$, respectively, $P=1.0$ ). Low-dose scans reconstructed with iDose level 4 had lower image noise compared to low-dose scans reconstructed with FBP $(P<0.001)$ (Table 1, Fig. 4).

PHV artifact volumes

Mean hypo- and hyperdense artifact volumes for different reconstruction algorithms are shown in Table 2 and classified per PHV type and

Table 1 Mean image noise measured in the plexiglass (PMMA) of the perfusion chamber for different reconstruction algorithms

\begin{tabular}{ll}
\hline Reconstruction algorithm & $\begin{array}{l}\text { Image noise PMMA } \\
\text { Mean } \pm \text { SD }\end{array}$ \\
\hline $120 \mathrm{kV} 600 \mathrm{mAs}$ FBP & $16.3 \pm 1.6$ \\
$120 \mathrm{kV} 300 \mathrm{mAs}$ FBP & $23.2 \pm 2.3$ \\
$120 \mathrm{kV} \mathrm{300} \mathrm{mAs} \mathrm{IR} \mathrm{level} \mathrm{4}$ & $16.5 \pm 1.7$ \\
\hline
\end{tabular}

FBP filtered back projection, $I R$ iterative reconstruction algorithm

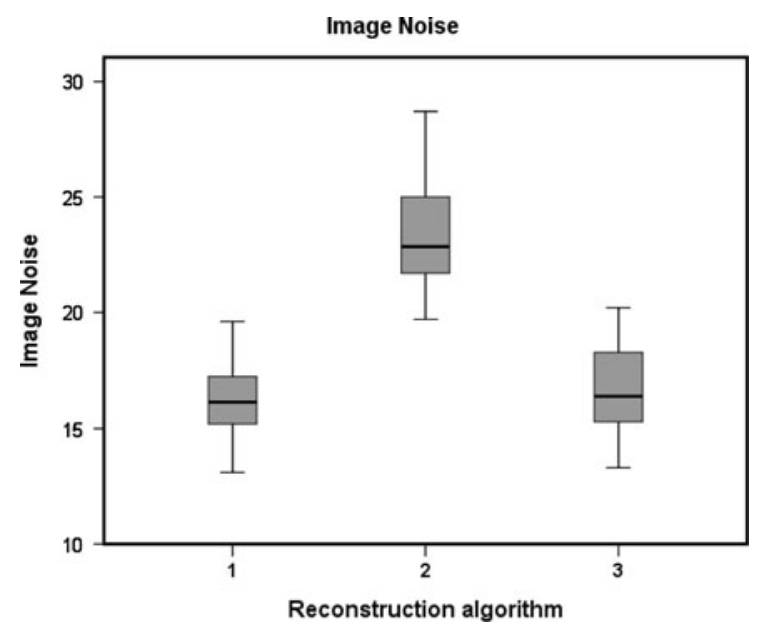

Fig. 4 Boxplots of image noise for different reconstruction algorithms. $1120 \mathrm{kV}, 600 \mathrm{mAs}$ FBP reconstruction $2120 \mathrm{kV}$, 300 mAs FBP reconstruction 3120 kV, 300 mAs iDose level 4 
Table 2 Mean artifact volumes $\left(\mathrm{mm}^{3}\right)$ for hypo- and hyperdense PHV artifacts for different reconstruction algorithms (all PHVs combined diastolic and systolic phases)

\begin{tabular}{lll}
\hline $\begin{array}{l}\text { Reconstruction } \\
\text { algorithm }\end{array}$ & $\begin{array}{l}\text { Hypodense } \\
\text { artifacts } \\
\text { Mean } \pm \text { SD }\end{array}$ & $\begin{array}{l}\text { Hyperdense } \\
\text { artifacts } \\
\text { Mean } \pm \text { SD }\end{array}$ \\
\hline $120 \mathrm{kV} 600$ mAs FBP & $1,235 \pm 731$ & $5,346 \pm 1,677$ \\
$120 \mathrm{kV} \mathrm{300} \mathrm{mAs} \mathrm{FBP}$ & $2,405 \pm 1,051$ & $6,877 \pm 1,849$ \\
$\begin{array}{l}120 \mathrm{kV} \mathrm{300} \text { mAs } \\
\text { (IR level 4) }\end{array}$ & $1,218 \pm 683$ & $5,333 \pm 1,663$ \\
\hline
\end{tabular}

FBP filtered back projection, IR iterative reconstruction algorithm

reconstruction phase in Table 3. Boxplots of mean hypodense and hyperdense PHV artifact volumes are shown in Fig. 5a and b. Repeated measures analyses were performed for each PHV type. For all PHV types, hypo- and hyperdense artifact volumes were significantly higher at low-dose scans compared to high dose scans reconstructed with FBP $(P<0.001)$.

For all PHV types, hypodense artifact volumes were similar (ON-X PHVs $P=0.55$ and other PHVs $P=1.0$, respectively) for the high-dose scans reconstructed with FBP when compared to low-dose scans reconstructed with IR. Hyperdense artifacts were similar for the high-dose scans reconstructed with FBP when compared to low-dose scans reconstructed with IR (St Jude $P=0.95$, all other valves $P=1.0$ ).

Hypodense artifact volumes were significantly smaller in the diastolic (closed valve) phase compared to the systolic (opened valve) phase for St Jude PHVs $(P=0.003)$; Medtronic Hall PHVs $(P<0.001)$; Carbomedics PHVs $(P<0.001)$ and ON-X PHVs $(P<0.001)$ (Table 3). Hyperdense artifact volumes were also significant smaller in the diastolic phase as opposed to the systolic phase for Medtronic Hall PHVs $(P<0.001)$, Carbomedics PHVs $(P=0.002)$, and ON-X PHVs $(P<0.001)$. The St Jude PHV demonstrated similar hyperdense artifacts $(P=0.074)$.

\section{Discussion}

The principal finding of this study is that iterative reconstruction allows different PHVs in a pulsatile in vitro model to be visualized with similar image noise and artifacts at a considerably reduced radiation dose $\left(50 \% \mathrm{CTDI}_{\mathrm{vol}}\right.$ and DLP) compared to conventional acquisitions reconstructed with FBP.

In a recent editorial [8] Fleischman and Boas provided an insightful summary of the history and principles underlying iterative reconstruction. They explain that reconstruction of raw CT data is currently performed using the FBP algorithm on virtually all commercially available CT systems [8]. The principle of FBP relies on the exact mathematical relationship between the measured X-ray attenuation in the projection data and the pixel values in the corresponding image. FPB assumes exact data. In reality, however, projection data from the CT scanner are

Table 3 Hypodense and hyperdense artifacts in systolic and diastolic phase for each PHV type and reconstruction algorithm

\begin{tabular}{|c|c|c|c|c|c|}
\hline \multirow[t]{2}{*}{ Reconstruction algorithm } & \multirow[t]{2}{*}{ Reconstruction algorithm } & \multicolumn{2}{|c|}{$\begin{array}{l}\text { Hypodense artifacts } \\
\text { Mean } \pm \text { SD }\end{array}$} & \multicolumn{2}{|c|}{$\begin{array}{l}\text { Hyperdense artifacts } \\
\text { Mean } \pm \text { SD }\end{array}$} \\
\hline & & Systolic & Diastolic & Systolic & Diastolic \\
\hline \multirow[t]{3}{*}{ St Jude Bileaflet PHV } & $120 \mathrm{kV}, 600 \mathrm{mAs} F B P$ & $1,829 \pm 488$ & $1,572 \pm 341$ & $7,170 \pm 352$ & $7,180 \pm 492$ \\
\hline & $120 \mathrm{kV}, 300 \mathrm{mAs}$ FBP & $2,994 \pm 275$ & $2,436 \pm 299$ & $8,770 \pm 254$ & $8,580 \pm 355$ \\
\hline & $120 \mathrm{kV}, 300 \mathrm{mAs}$ IR level 4 & $1,890 \pm 208$ & $1,484 \pm 208$ & $7,120 \pm 253$ & $6,920 \pm 294$ \\
\hline \multirow[t]{3}{*}{ Carbomedics Bileaflet PHV } & $120 \mathrm{kV}, 600 \mathrm{mAs}$ FBP & $630 \pm 137$ & $342 \pm 43$ & $3,010 \pm 175$ & $2,836 \pm 125$ \\
\hline & $120 \mathrm{kV}, 300 \mathrm{mAs}$ FBP & $1,582 \pm 232$ & $1,062 \pm 147$ & $4,281 \pm 318$ & $3,992 \pm 153$ \\
\hline & $120 \mathrm{kV}, 300 \mathrm{mAs}$ IR level 4 & $683 \pm 167$ & $322 \pm 56$ & $3,051 \pm 264$ & $2,746 \pm 120$ \\
\hline \multirow[t]{3}{*}{ ON-X Bileaflet PHV } & $120 \mathrm{kV}, 600 \mathrm{mAs}$ FBP & $1,237 \pm 216$ & $790 \pm 73$ & $5,292 \pm 178$ & $5,052 \pm 57$ \\
\hline & $120 \mathrm{kV}, 300 \mathrm{mAs}$ FBP & $2,265 \pm 267$ & $1,637 \pm 127$ & $6,752 \pm 179$ & $6,582 \pm 107$ \\
\hline & $120 \mathrm{kV}, 300 \mathrm{mAs}$ IR level 4 & $1,198 \pm 138$ & $716 \pm 45$ & $5,282 \pm 171$ & $5,032 \pm 57$ \\
\hline \multirow[t]{3}{*}{ Medtronic Hall tilting disc PHV } & $120 \mathrm{kV}, 600 \mathrm{mAs}$ FBP & $2,575 \pm 433$ & $905 \pm 116$ & $6,880 \pm 1,001$ & $5,350 \pm 196$ \\
\hline & $120 \mathrm{kV}, 300 \mathrm{mAs}$ FBP & $4,534 \pm 564$ & $2,729 \pm 283$ & $8,910 \pm 928$ & $7,150 \pm 268$ \\
\hline & $120 \mathrm{kV}, 300 \mathrm{mAs}$ IR level 4 & $2,385 \pm 489$ & $1,068 \pm 325$ & $7,010 \pm 936$ & $5,500 \pm 542$ \\
\hline
\end{tabular}



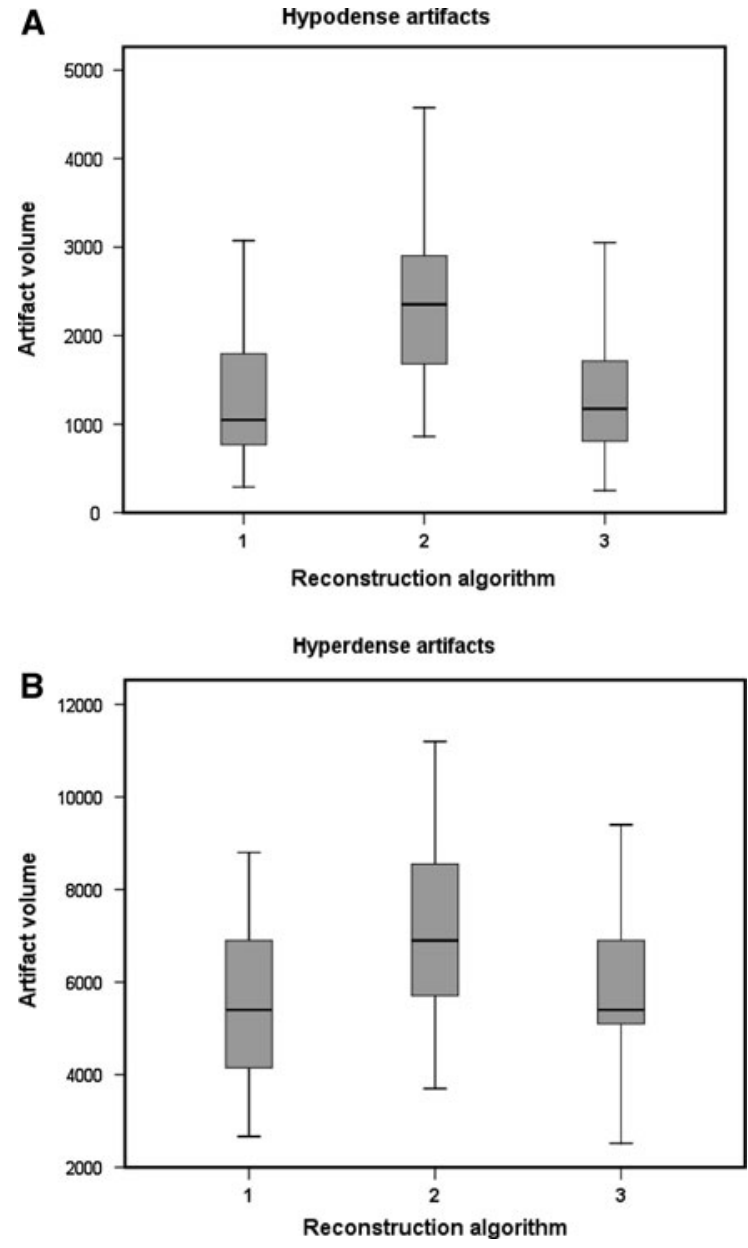

Fig. 5 Boxplots of hypodense (a) and hyperdense (b) PHV artifacts for different reconstruction algorithms. $1120 \mathrm{kV}$, $600 \mathrm{mAs}$ FBP reconstruction, $2120 \mathrm{kV}, 300 \mathrm{mAs}$ FBP reconstruction, $3120 \mathrm{kV}, 300 \mathrm{mAs}$ iDose level 4

noisy. The filter used in FBP increases image noise [8]. Iterative reconstruction utilizes statistical models to improve signal to noise with each iteration. The main advantage of iterative reconstruction compared to FBP is the reduced image noise. Furthermore, iterative reconstruction reduces metal streak artifacts $[8,16]$.

Initial clinical results with iterative reconstruction are encouraging. Iterative reconstruction algorithms have been shown to decrease image noise and improve image quality of coronary segments in patients that underwent cardiac CTA for clinical indications at lower radiation dose $[11,12]$. Nevertheless, little is known about the application of iterative reconstruction algorithms in MDCT imaging of PHVs. We evaluated the effect of a novel IR algorithm (iDose,
Philips Medical Systems, Best, The Netherlands) that performs iterative processing in both the projection and image domains on the image noise at a considerable lower radiation dose. Additionally, the effect of IR on the artifacts typically associated with MDCT imaging of PHV was evaluated.

The amount of noise in CT images depends on several factors: section collimation, acquisition time, and tube current [14]. Theoretically a decrease in tube current by a factor 2 leads to an increase in image noise by the square root of 2 (1.41), given that all other scanning parameters are kept constant [14]. By reconstructing images acquired with half the tube current using iDose level 4 IR, we expected, and found, noise to be reduced to the level of the initial tube current setting (Table 1). When low dose scans were reconstructed with iDose level 4 , mean image noise was again similar to the high-dose scans (Table 1). The $50 \%$ radiation dose reduction (CTDI${ }_{\text {vol }} / \mathrm{DLP} 35.3 / 473.4 \mathrm{mGy}$ vs. $17.7 / 236.5 \mathrm{mGy}$ ) is mainly compensated for by the improvement of spatial resolution due to the denoising properties of the iterative reconstruction algorithm (Fig. 6). Hypo- and hyperdense artifacts were comparable between high dose scans with FBP and low dose scans reconstructed with iDose level 4 IR (Fig. 7). IR performed similar in the systolic and diastolic phase for the reduction of PHV artifacts (Table 3).

\section{Limitations}

This study has limitations. First, the study was performed in an in-vitro model. In this model, the normal annular motion present in the human heart, which may induce additional artifacts, is absent. However, strictly standardized conditions are essential for the comparison between different reconstruction algorithms. Second, no contrast medium was administered. The usage of diluted contrast would have represented a more realistic situation. However, in patients contrast timing, amount and enhancement may differ widely between centers. We used water as perfusate to standardize the experimental set-up. Furthermore we do not expect the artifacts generated by the PHV itself to be influenced by contrast material. Third, no PHV pathology was imaged in this in vitro model. Therefore, diagnostic accuracy cannot be verified. Artifacts are the main reasons for nonassessable PHVs. These artifacts were similar for the 

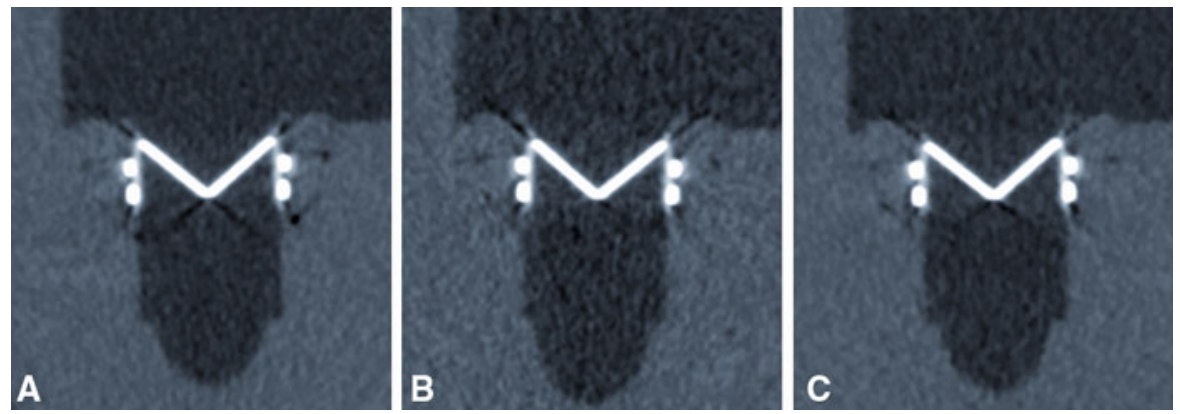

Fig. 6 MDCT image of ON-X Bileaflet PHV. a Frontal plane 120 kV, 600 mAs reconstructed with FBP, b Frontal plane 120 kV 300 mAs reconstructed with FBP, c Frontal plane 120 kV 300 mAs reconstructed with iDose level 4
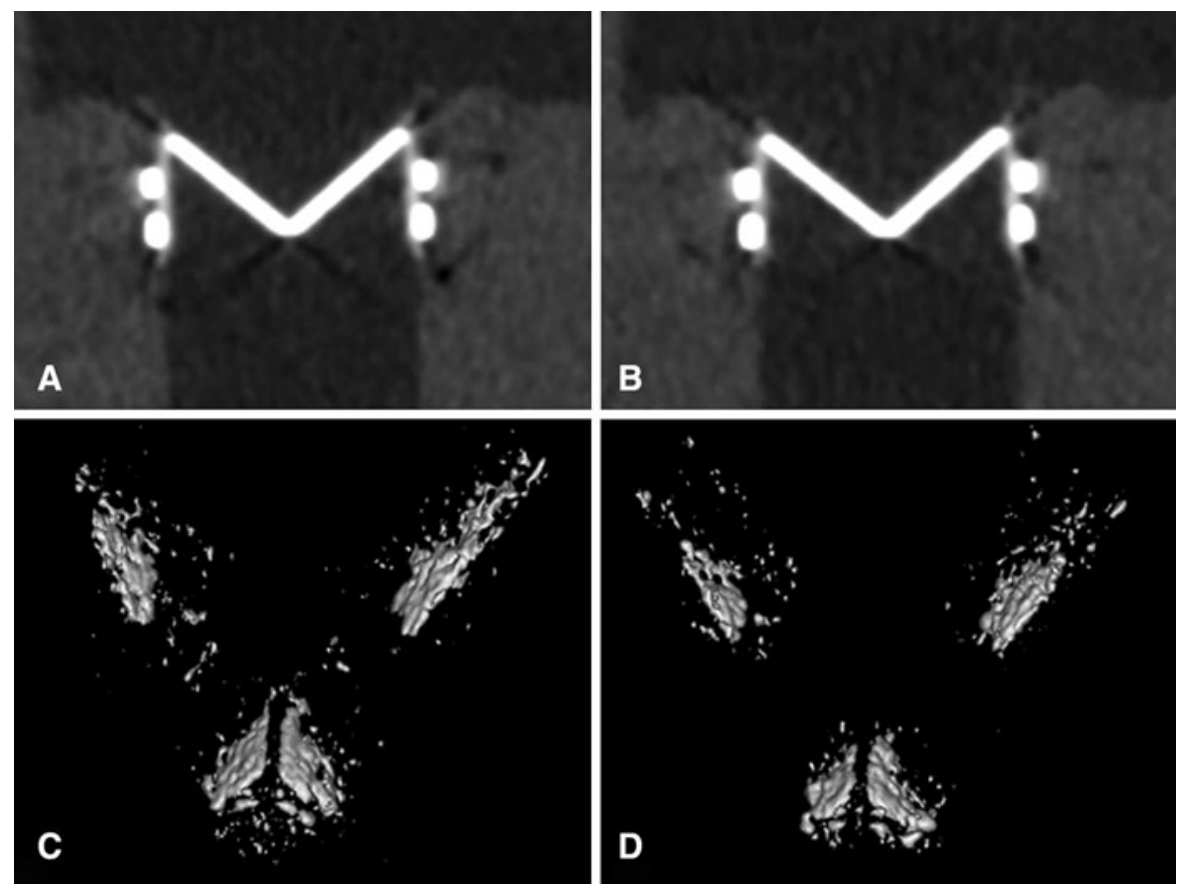

Fig. 7 MDCT image of ON-X Bileaflet PHV. a Frontal plane $120 \mathrm{kV}, 600 \mathrm{mAs}$ reconstructed with FBP, b Frontal plane $120 \mathrm{kV}, 300 \mathrm{mAs}$ reconstructed with iDose level 4, c Frontal

plane with $3 \mathrm{D}$ volume PHV hypodense artifacts $(120 \mathrm{kV}$, $600 \mathrm{mAs}$, BFP), d Frontal plane with 3D volume PHV hypodense artifacts $(120 \mathrm{kV}, 300 \mathrm{mAs}$, iDose level 4)

iterative reconstructions compared to the high-dose scans. Therefore, our assumption is that pathology can be equally well detected. Fourth, because hypodense artifact volumes ( $\leq 50 \mathrm{HU}$ filter) did not only include hypodense PHV artifacts but also image noise, we cannot rule out an influence on the results. However, the appearance of hypodense artifacts was similar for high dose scans reconstructed with FBP and low dose scans reconstructed with IR (Fig. 7). Fifth, we scanned

only with one tube voltage setting $(120 \mathrm{kV})$. In our institution, $120 \mathrm{kV}$ is commonly used for PHV CT imaging. Experiments with different $\mathrm{kV}$ settings (100, $140 \mathrm{kV}$ ) would be interesting to optimize PHV CT scan protocols. Sixth, image quality was not assessed. Continuous outcome measures (3D artifact volumes and image noise) were preferred to quantify the effect of iterative reconstruction algorithms on image quality because of the more objective nature of these outcome 
measures. Further clinical evaluation in patients with PHVs is required to determine if PHV imaging with IR can replace standard FBP at a lower radiation dose. In prospective clinical studies, other patient related factors (e.g. body mass index) which can influence CT image quality have to be taken into account. Besides the novel IR algorithms other dose reducing techniques (e.g. prospective ECG triggering) have to be evaluated for PHV imaging. At present, retrospectively ECG-gated scanning remains essential to obtain dynamic information in both systolic and diastolic phases which is required for adequate leaflet motion assessment. Sixth, only one heart rate $(60 \mathrm{bpm})$ was explored. Higher heart rates and arrhythmia may influence PHV CT image quality. Wide area detector CT scanners (256 slice or higher) enable prospective data acquisition in one or only several heart beats [17, 18]. The more efficient radiation use in prospective scanning compared to retrospective scanning and the lower or 0 pitch may influence PHV generated artifacts. On the other hand this may improve image quality compared to 64 slice CT scanning. High-pitch spiral scanning is an interesting alternative for image acquisition because of the decreased radiation exposure $[19,20]$. Low heart rates $(<60 \mathrm{bpm})$ are required to allow high-pitch spiral scanning. However, most patients with suspected PHV dysfunction have high heart rates or rhythm disorders and contraindications for $\beta$-blockers which preclude high-pitch spiral scanning. In further prospective studies, a multivariate approach including different heart rates and different scanner types would be an interesting research topic. Finally, we investigated just one level of iterative reconstruction. It is possible that lower or higher levels of IR could have led to different results.

\section{Conclusion}

In conclusion, MDCT imaging using iterative reconstruction enables in vitro imaging of different PHV types at 50\% lower radiation dose with similar image noise and hypo- and hyperdense PHV artifacts.

Acknowledgments We would like to thank Karin Rijnbach, Multimedia specialist, Department of Digital Photography, University Medical Center Utrecht for her help with edition of the figures. The present study was supported by a grant of The Netherlands Heart Foundation [Grant number 2009B014].

\section{Conflict of interest None.}

Open Access This article is distributed under the terms of the Creative Commons Attribution Noncommercial License which permits any noncommercial use, distribution, and reproduction in any medium, provided the original author(s) and source are credited.

\section{References}

1. Tsai IC, Lin YK, Chang Y et al (2009) Correctness of multidetector-row computed tomography for diagnosing mechanical prosthetic heart valve disorders using operative findings as a gold standard. Eur Radiol 19:857-867

2. Symersky P, Budde RP, de Mol BA et al (2009) Comparison of multidetector-row computed tomography to echocardiography and fluoroscopy for evaluation of patients with mechanical prosthetic valve obstruction. Am J Cardiol 104:1128-1134

3. Konen E, Goitein O, Feinberg MS et al (2008) The role of ECG-gated MDCT in the evaluation of aortic and mitral mechanical valves: initial experience. Am J Roentgenol 191:26-31

4. Feuchtner GM, Stolzmann P, Dichtl W et al (2009) Multislice computed tomography in infective endocarditis: comparison with transesophageal echocardiography and intraoperative findings. J Am Coll Cardiol 53:436-444

5. Habets J, Budde RP, Symersky P et al (2011) Diagnostic evaluation of left-sided prosthetic heart valve dysfunction. Nat Rev Cardiol 8:466-478

6. Habets J, Symersky P, van Herwerden LA et al (2011) Prosthetic heart valve assessment with multidetector-row CT: imaging characteristics of 91 valves in 83 patients. Eur Radiol 21:1390-1396

7. Chenot F, Montant P, Goffinet C et al (2010) Evaluation of anatomic valve opening and leaflet morphology in aortic valve bioprosthesis by using multidetector CT: comparison with transthoracic echocardiography. Radiology 255:377-385

8. Fleischmann D, Boas FE (2011) Computed tomographyold ideas and new technology. Eur Radiol 21:510-517

9. Lange K, Carson R (1984) EM reconstruction algorithms for emission and transmission tomography. J Comput Assist Tomogr 8:306-316

10. Shepp LA, Vardi Y (1982) Maximum likelihood reconstruction for emission tomography. IEEE Trans Med Imaging 1:113-122

11. Leipsic J, LaBounty TM, Heilbron B et al (2010) Estimated radiation dose reduction using adaptive statistical iterative reconstruction in coronary CT angiography: the ERASIR study. Am J Roentgenol 195:655-660

12. Leipsic J, LaBounty TM, Heilbron B et al (2010) Adaptive statistical iterative reconstruction: assessment of image noise and image quality in coronary CT angiography. Am J Roentgenol 195:649-654

13. Symersky P, Budde RPJ, Prokop M et al (2011) Multidetector-row computed tomography imaging characteristics of mechanical prosthetic valves. J Heart Valve Dis 20:216-222 
14. Prokop M (2003) Radiation dose and image quality. In: Prokop M, Galanski M (eds) Spiral and multislide computed tomography of the body. Thieme, Stuttgart, pp 143-144

15. van der Schaaf I, van Leeuwen M, Vlassenbroek A et al (2006) Minimizing clip artifacts in multi CT angiography of clipped patients. Am J Neuroradiol 27:60-66

16. Boas FE, Fleischmann D (2011) Evaluation of two iterative techniques for reducing metal artifacts in computed tomography. Radiology 259:894-902

17. Rybicki FJ, Otero HJ, Steigner ML et al (2008) Initial evaluation of coronary images from 320-detector row computed tomography. Int J Cardiovasc Imaging 24:535-546
18. Weigold WG, Olsezewski ME, Walker MJ (2009) Lowdose prospectively gated 256-slice coronary computed tomographic angiography. Int $\mathrm{J}$ Cardiovasc Imaging 25:217-230

19. Achenbach S, Marwan M, Ropers D et al (2010) Coronary computed tomography angiography with a consistent dose below $1 \mathrm{mSv}$ using prospectively electrocardiogram-triggered high-pitch spiral acquisition. Eur Heart 31:340-346

20. Achenbach S, Marwan M, Schepis T et al (2009) High-pitch spiral acquisition: a new scan mode for coronary CT angiography. J Cardiovasc Comput Tomogr 3:117-121 